\title{
Ocorrência e danos causados por Pygiopachymerus lineola (Chevrolat, 1971) (Coleoptera: Chrysomelidae: Bruchinae) em frutos de Cassia fistula $\mathbf{L}$. (Caesalpiniaceae: Caesalpinioideae) em Maceió, Brasil. ${ }^{(1)}$
}

\author{
EMMELYNE KETLLEN SOARES LUZ DA COSTA ${ }^{(2,3)}$; IRACILDA MARIA DE MOURA LIMA ${ }^{(4)}$ \\ e THAIS RANIELLE SOUZA DE OLIVEIRA ${ }^{(5)}$
}

\begin{abstract}
RESUMO
Cassia fistula L. (Caesalpiniaceae: Caesalpinioideae) é uma planta ornamental conhecida pelos nomes populares de canafístula, cássia-imperial ou cássia-chuva-de-ouro. Originária da Ásia, também se encontra nos continentes americano e africano, sendo uma das mais utilizadas em projetos de paisagismo, produção de mudas e de arborização urbana no Brasil pela beleza de suas grandes inflorescências pendentes amarelas. Sabe-se que muitas espécies arbóreas têm suas sementes significativamente danificadas por vários grupos de insetos, destacando-se os carunchos (Coleoptera: Chrysomelidae: Bruchinae), que inviabilizam as sementes e consequentemente a produção das mudas. O objetivo deste estudo foi determinar os agentes de controle natural das sementes de $C$. fistula (predadores de sementes), através da determinação da espécie envolvida na predação, a razão de viabilidade dos ovos e a descrição de alguns aspectos de funções biológicas (alimentação e reprodução). O trabalho foi conduzido no Laboratório de Entomologia, Museu de História Natural, Universidade Federal de Alagoas, Maceió. Foram recolhidas 20 vagens de $C$. fistula de cada planta em três bairros de Maceió. As vagens estavam completamente desenvolvidas, secas e com ovos em sua superfície. Os insetos foram acondicionados em álcool $70 \%$ e identificados em nível de espécie. Na amostra A, 89\% das vagens apresentaram indícios de predação; em B, 95\%; e em C, 88\%. O número de orifícios / vagem nas amostras A, B e C foi respectivamente: 3 a 26, 1 a 19 e 1 a 18. O número de predadores / vagem foi: em A de 10 a 26, em B de 1 a 8 , e em C de 2 a 14. Para as três amostras observou-se correlação positiva alta $(\mathrm{r}>0,8)$, revelando que o número de orifícios é muito aproximado ao número de insetos que saem das vagens. Quanto à viabilidade dos ovos verificou-se: em A, 84,2\% de vagens com ovos viáveis; em B, $100 \%$; e em C, 83,3\%.
\end{abstract}

Palavras-chave: predadores de sementes, bruquíneos, oviposição.

\begin{abstract}
Occurrence and damage caused by Pygiopachymerus lineola (Chevrolat, 1971) (Coleoptera: Chrysomelidae: Bruchinae) in Cassia fistula L. (Caesalpiniaceae: Caesalpinioideae) fruits in Maceió, Brazil

Cassia fistula L. (Caesalpiniaceae: Caesalpinioideae) is an ornamental plant popularly known by golden-shower, Indianlaburnum or purging-cassia; in Brazil it is named canafístula, cássia-imperial or cássia-chuva-de-ouro. Native to Asia, it also occurs in the Americas and Africa, being one of the most used in landscaping projects, seedling production and urban forestry in Brazil by the large yellow hanging beauty flowers. It is known that many tree species have their seeds significantly damaged by several insect groups, especially seed beetles (Coleoptera: Chrysomelidae: Bruchinae), that become the seeds unviable and consequently diminishing the seedlings production. The objective of this study was to determine the natural control agents of the $C$. fistula seeds (seed predators) through the determination of species involved in the predation, the ratio of egg viability and description the some biological functions aspects (feeding and reproduction). The work was conducted at the Entomology Laboratory, Natural History Museum, Federal University of Alagoas, Maceió. Were collected from each plant of $C$. fistula 20 fully developed pods and with dried eggs on its surface. The insects were placed in $70 \%$ alcohol and identified to the species level. In the sample A, $89 \%$ of string beans showed the predation phenomenon; in B, $95 \%$; and C, $88 \%$. The hole numbers / pod in samples A, B and C were respectively: 3-26, 1-19 and 1 to 18 . The predator numbers / pod were: in A, 10 to 26; in B, 1-8; and in C, 2 to 14. The three samples showed high positive correlation ( $r>$ 0.8 ), revealing that the number of holes is very close to the number of insects that emerged from the pods. Viable eggs occurred in the $84.2 \%$ of pods in $\mathrm{A}, 100 \%$ in $\mathrm{B}$, and $83.3 \%$ in $\mathrm{C}$.
\end{abstract}

Keywords: seed predators, bruchines, oviposition.

\section{INTRODUÇÃO}

Cassia fistula L. (Caesalpiniaceae: Caesalpinioideae), conhecida pelos nomes populares de canafístula, cássiaimperial, e principalmente, cássia-chuva-de-ouro, apesar de exótica (originária da Ásia), encontra-se adaptada ao clima do Nordeste brasileiro, sendo muito utilizada na ar- borização urbana principalmente em jardins, parques, praças e calçadas pela beleza de suas grandes inflorescências pendentes amarelas que desabrocham ao longo dos meses de setembro a fevereiro (LORENZI, 2001).

A planta é uma árvore de grande porte e muito resistente que produz uma grande quantidade de inflorescências amarelas e conserva a folhagem por ocasião dos verões, mesmo nas piores secas (BRAGA, 1976).

\footnotetext{
(1) Trabalho recebido para publicação em 30/06/2011 e aprovado em 26/05/2014;

(2) Colaboradora Iniciação Científica CNPq/UFAL/FAPEAL 2006-2007. emmelyne@gmail.com)

(3) Professora Adjunta, Setor de Biodiversidade e Ecologia- do Instituto de Ciências Biológica se da Saúde ICBS-UFAL. iralcildalimae@gmail.com

(4) Aluna de doutorado do Programa de Pós-Graduação em Ciências Biológicas (Zoologia) UFPB. thaisranielle@gmail.com

(5) Professora Adjunta, Departamento de Zoologia, Universidade Federal do Paraná. stra@ufpr.br
} 
No entanto, tem se verificado, por parte dos produtores de mudas, uma taxa de germinação muito baixa, às vezes chegando a zero. Esse problema também é constatado em torno das plantas-mãe, onde raramente se observa plântulas jovens próximas da copa. Sabe-se que muitas espécies arbóreas têm suas sementes significativamente danificadas por vários grupos de insetos (CARVALHO E FIGUEIRA, 1999; SARI et al. 2005), destacando-se os carunchos (Coleoptera: Chrysomelidae: Bruchinae).

Os insetos adultos depositam os ovos sobre a superfície de vagens. As larvas eclodem, perfuram a casca da vagem e penetram nas sementes permanecendo no seu interior, consumindo os cotilédones à medida que crescem (JANZEN, 1971; RICKFLES, 2003), inviabilizando as sementes e consequentemente a produção das mudas.

Assim, determinar a importância bioecológica de espécies vegetais de uso paisagístico e que se desenvolvem nas áreas urbanas tem grande importância quando é necessário se estabelecerem, em projetos de parques e jardins, justificativas para a escolha de plantas visando à composição da paisagem (DANTAS e SOUZA, 2004; LIMA et al., 2007; PEREIRA et al., 2010), o que consequentemente se relaciona à facilidade de obtenção de mudas. Sari et al. (2005) e Mattiuz et al. (2006) ressaltaram a importância de pesquisas relacionadas à ocorrência de insetos e seus danos causados em plantas ornamentais utilizadas também em áreas urbanas na atualidade.

Neste contexto, o objetivo deste estudo foi determinar os agentes de controle natural das sementes de $C$. fistula (predadores de sementes) através da determinação da espécie envolvida com a predação, a razão de viabilidade dos ovos e a descrição de alguns aspectos de funções biológicas (alimentação e reprodução).

\section{MATERIAIS E MÉTODOS}

O trabalho foi conduzido no Laboratório de Entomologia do Museu de História Natural da Universidade Federal de Alagoas situado o município de Maceió, Estado de Alagoas, de $1^{\circ}$ junho a dezembro de 2006, sob condições ambientais médias de $27,8^{\circ} \mathrm{C}$ e $60,7 \%$ de umidade relativa do ar (parâmetros determinados a partir dos dados de registro diário em termo-higrômetro).

As amostras foram coletadas em 3 bairros de Maceió: Jatiúca (A), Farol (B) e Cidade Universitária (C). Para coleta das vagens foi utilizado equipamento de poda, nas vagens que estavam em galhos mais altos, e a coleta manual em três árvores de C. fistula (Figura 1.a), sendo recolhidas, de cada planta, 20 vagens completamente desenvolvidas e secas. Algumas possuíam ovos circulares e achatados depositados na superfície. a)

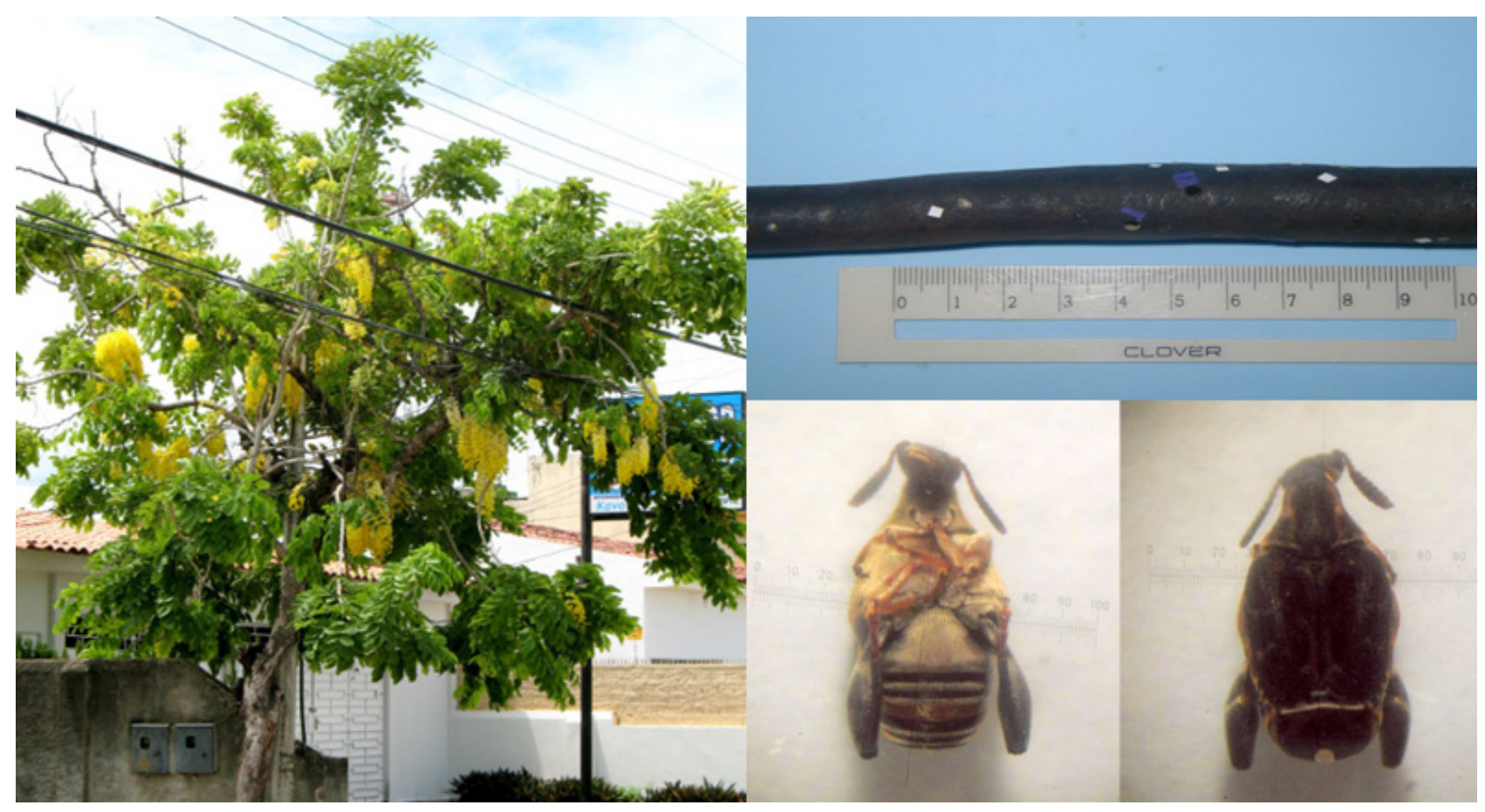

Figura 1. (a) Cassia fistula L. (Leguminosae: Caesalpinoideae) no bairro do Farol, Maceió-Al. Foto: Allen Kinsen. (b) Vagem de C. fistula com etiquetas adesivas para marcação dos ovos e orifícios causados por (c) Pygiopachymerus lineola (Chevrolat, 1871) (Coleoptera: Chrysomelidae). Foto: Maria Dulce Leão Marciano.

Figure 1. (a) Cassia fistula L. (Leguminosae: Caesalpinoideae) in the suburb of Farol, Maceió-Al. Photo: Allen Kinsen. (b) pod C. fistula with adhesive labels for marking eggs and holes caused by (c) Pygiopachymerus lineola (Chevrolat, 1871) (Coleoptera: Chrysomelidae). Photo: Dulce Maria Leon Marciano. 
Foram fixadas nos frutos etiquetas adesivas cortadas em pequenos quadrados $(0,4 \mathrm{~cm})$ para marcação e contagem dos ovos. A observação foi realizada em estereomicroscópio para facilitar a diferenciação dos ovos viáveis (com aparência transparente) e não viáveis (aparência leitosa) (Figura 1.b).

As 60 vagens foram armazenadas individualmente sem sofrerem quebras, para não alterar o possível desenvolvimento dos insetos, em recipientes de plástico feitos com garrafas PET $^{\circledR}$ (Polietileno tereftalato) isolados com fita adesiva transparente, cobertos com organdi fixado com liga de borracha sendo posteriormente etiquetados.

Diariamente, à medida que os predadores emergiam das vagens, os orifícios eram marcados com auxílio de etiquetas adesivas vermelhas, cortadas em quadrados e coladas na superfície dos frutos. Todos os dados necessários foram registrados em etiquetas, planilhas de acompanhamento, sendo feitos registros fotográficos (Figura 1.c). Depois da saída das vagens, os insetos foram acondicionados em solução de álcool 70\% em tubos eppendorf, e outra parte foi montada segundo Almeida et al., (1998) (montagem dupla), sendo depositados na Coleção Entomológica, Museu de História Natural, Universidade Federal de Alagoas, Maceió, e Coleção Entomológica Padre Jesus Santiago Moure, Universidade Federal do Paraná, Curitiba.

A planta foi depositada na coleção de plantas do Herbário situado no Instituto do Meio Ambiente (IMA) e a chave de identificação utilizada foi de acordo com Lorenzi (2007). Os procedimentos adotados foram baseados na obra de Alves e Pavani (1991).

Para a análise dos dados coletados durante o estudo como a quantidade de insetos por amostra, razão de viabilidade dos ovos e escape, foram utilizadas as estatísticas descritivas, medidas de tendência central (média, moda e mediana) e de dispersão (coeficiente de variação, amplitude, intervalo de variação e intervalo de confiança). O coeficiente de variação foi analisado segundo Born e Lima (2005), está enquadrado em quatro classes: I, homogêneo $(<10 \%)$; II, relativamente homogêneo (10-20\%); III, relativamente heterogêneo (20-30\%); e IV, heterogêneo (> $30 \%$ ). Foi utilizada a correlação de Pearson para avaliar a relação entre número de orifícios e o número de insetos.

\section{RESULTADOS E DISCUSSÃO}

A percentagem de predação nas vagens de $C$. fistula foi de $89 \%$ em A, $95,0 \%$ em B e $88 \%$ em C, indicando que em $B$ houve maior percentual em relação aos demais que obtiveram valores parecidos. Investigando a ação predatória em frutos de Apuleia leiocarpa (VOGEL) J. F. Macbr. (Caesalpinioideae), Santos et al. (1989), relataram que $25 \%$ dos frutos estudados foram danificados enquanto que Loureiro et al. (2004) obtiveram um valor inferior correspondendo a $0,6 \%$ dos frutos. Os dados obtidos neste estudo representam, portanto, alta taxa predação.

Em relação ao número de orifícios por vagem nas amostras A, B e C, os coeficientes de variação foram $66,2 \%$, $77,4 \%$ e $81 \%$ (Tabela 1 ). Portanto, observando os escapes de $P$. lineola nas amostras A, B e C os coeficientes de variação foram $57,0 \%, 66,6 \%$ e $54,0 \%$. Assim sendo esses valores confirmam que os dados referentes às três amostras são heterogêneos, pois ultrapassam o valor de $30 \%$.

Tabela 1. Estatísticas descritivas da predação de vagens de Cassia fistula L. (Leguminosae: Caesalpinoideae) por Pygiopachymerus lineola (Chevrolat, 1871) (Coleoptera: Chrysomelidae) nas amostras A, B e C sob condições ambientais de $27,8^{\circ} \mathrm{C}$ e $60,7 \%$ de umidade relativa do ar.

Table 1. Descriptive statistics of predation in fruit of Cassia fistula L. (Leguminosae: Caesalpinoideae) by Pygiopachymerus lineola (Chevrolat, 1871) (Coleoptera: Chrysomelidae) in samples A, B and C under ambient conditions of $27.8^{\circ}$ $C$ and $60.7 \%$ of relative humidity.

\begin{tabular}{|c|c|c|c|c|c|}
\hline & AMOSTRA & Média $\mathbf{s} \pm(\mathbf{m})^{1}$ & $\begin{array}{c}\text { MODA/ } \\
\text { MEDIANA }\end{array}$ & $\begin{array}{l}\text { AMPLITUDE } \\
\text { TOTAL } \\
\text { (MIN-MÁX) }\end{array}$ & $\begin{array}{l}\text { CV } \\
(\%)\end{array}$ \\
\hline \multirow{3}{*}{$\begin{array}{c}\text { No. total de Orifícios/vagem na } \\
\text { Amostra }\end{array}$} & A & $12,8 \pm 2,1$ & $2 / 5$ & $23(3$ a 26$)$ & 66,2 \\
\hline & B & $7,3 \pm 1,2$ & 3 e $5 / 7$ & $18(1$ a 19$)$ & 77,4 \\
\hline & $\mathrm{C}$ & $7,0 \pm 1,5$ & 2 & $17(1$ a 18$)$ & 81,0 \\
\hline \multirow{3}{*}{$\begin{array}{c}\text { No. total de predadores/vagem } \\
\text { na Amostra }\end{array}$} & A & $16,7 \pm 0,2$ & $17 / 16,5$ & $23(10$ a 26$)$ & 57,0 \\
\hline & B & $4,5 \pm 1$ & 4 e $6 / 3$ & $7(1$ a 8$)$ & 66,6 \\
\hline & $\mathrm{C}$ & $8,1 \pm 1,6$ & amodal/8 & $12(2$ a 14$)$ & 54,0 \\
\hline
\end{tabular}


O número médio de $P$. lineola que saíram dos orifícios nas vagens de $C$. fistula somando os valores dos três locais de coleta podem ser visualizados na figura 2. Para as três amostras observou-se correlação positiva alta $(r>0,8)$, revelando que o número de orifícios é muito aproximado ao número de insetos que saem das vagens. Para as três localidades observou-se correlação positiva e alta $(r>0,8)$, o que revela que o número de orifícios é muito aproximado ao número de insetos que saem das vagens (Figura 3). Os valores de $\mathrm{r}$ para cada amostra são mostrados na tabela 2 .

Tabela 2. Coeficiente de correlação linear simples e parâmetros de ajuste de regressão linear do número de orifícios efetuados por Pygiopachymerus lineola (Chevrolat, 1871) para saída de vagens de Cassia fistula L. (Leguminosae: Caesalpinoideae) em três bairros do município de Maceió, Estado de Alagoas, no período de junho a dezembro de 2006.

Table 2. Correlation coefficient and simple linear adjustment parameters of linear regression of the number of holes made by Pygiopachymerus lineola (Chevrolat, 1871) to output fruit of Cassia fistula L. (Leguminosae: Caesalpinoideae) in three districts of the city of Maceió, Alagoas state, in the period from June to December 2006.

\begin{tabular}{|c|c|c|c|c|c|}
\hline \multicolumn{2}{|c|}{ LOCAL } & \multicolumn{1}{c|}{$\mathbf{r}^{\mathbf{1}}$} & \multicolumn{2}{c|}{$\mathbf{a}^{\mathbf{2}}$} & $\mathbf{b}^{\mathbf{3}}$ \\
\hline BAIRRO & Amostra & & & \\
\hline Jatiùca & $\mathbf{A}$ & 0,95477 & $2,6 \pm 2,0$ & $1,0 \pm 0,2$ & 6 \\
\hline Farol & $\mathbf{B}$ & 0,80696 & $-0,9 \pm 2,0$ & $1,2 \pm 0,3$ & 10 \\
\hline Cidade universitária & $\mathbf{C}$ & 0,94267 & $0,8 \pm 1,3$ & $1,0 \pm 0,2$ & 7 \\
\hline
\end{tabular}

${ }^{1} \mathrm{r}$ : coeficiente de correlação.

${ }^{2} a$ : intersecção em y.

${ }^{3} \mathrm{~b}$ : coeficiente angular da reta.

${ }^{4} n$ : número de medidas correlacionadas, em $Y=A+B . X\left(Y=n^{\circ}\right.$ de orifícios e $X=n^{\circ}$ de insetos $)$.

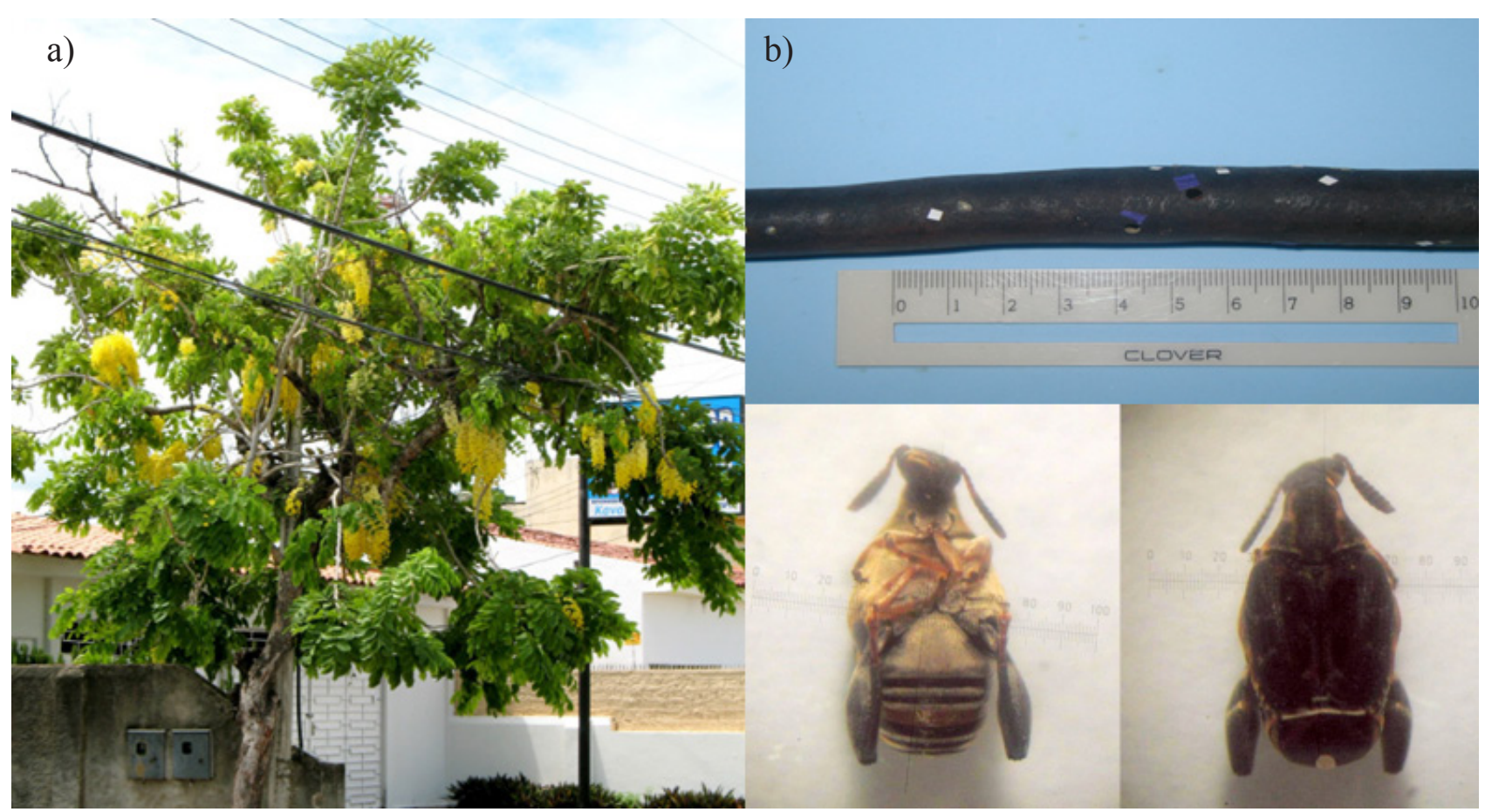

Figura 1. (a) Cassia fistula L. (Leguminosae: Caesalpinoideae) no bairro do Farol, Maceió-Al. Foto: Allen Kinsen. (b) Vagem de C. fistula com etiquetas adesivas para marcação dos ovos e orifícios causados por (c) Pygiopachymerus lineola (Chevrolat, 1871) (Coleoptera: Chrysomelidae). Foto: Maria Dulce Leão Marciano. Figure 1. (a) Cassia fistula L. (Leguminosae: Caesalpinoideae) in the suburb of Farol, Maceió-Al. Photo: Allen Kinsen. (b) pod C. fistula with adhesive labels for marking eggs and holes caused by (c) Pygiopachymerus lineola (Chevrolat, 1871) (Coleoptera: Chrysomelidae). Photo: Dulce Maria Leon Marciano. 


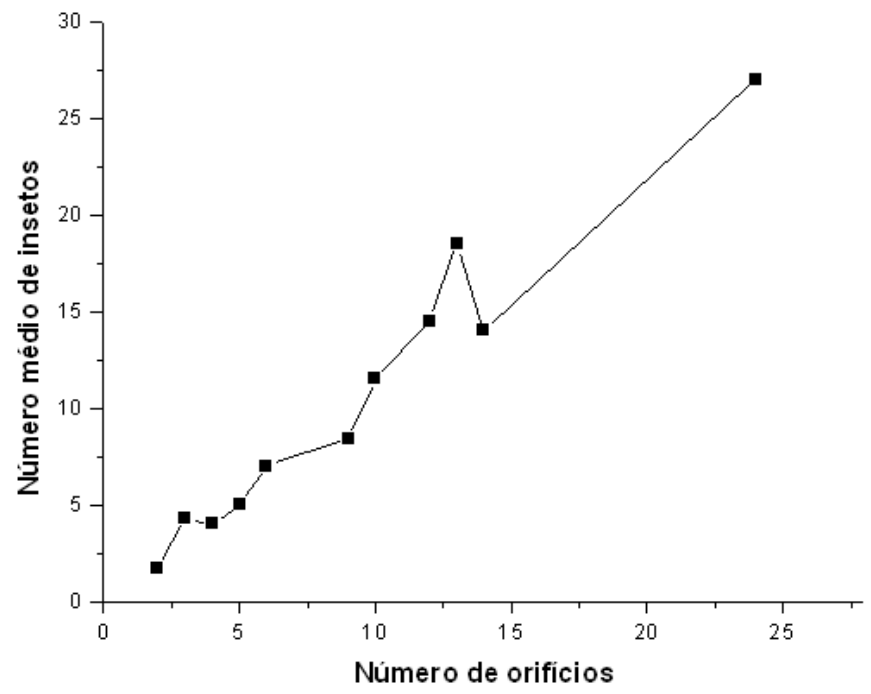

Figura 2. Número médio de exemplares de Pygiopachymerus lineola (Chevrolat, 1871) que podem sair dos orifícios construídos em vagens de Cassia fistula L. (Leguminosae: Caesalpinoideae) em três bairros do município de Maceió,

Estado de Alagoas, no período de junho a dezembro de 2006.

Figure 2. Average number of specimens of Pygiopachymerus lineola (Chevrolat, 1871) that can leave holes built in pods of Cassia fistula L. (Leguminosae: Caesalpinoideae) in three districts of the city of Maceió, Alagoas state, in the period from June to December 2006.

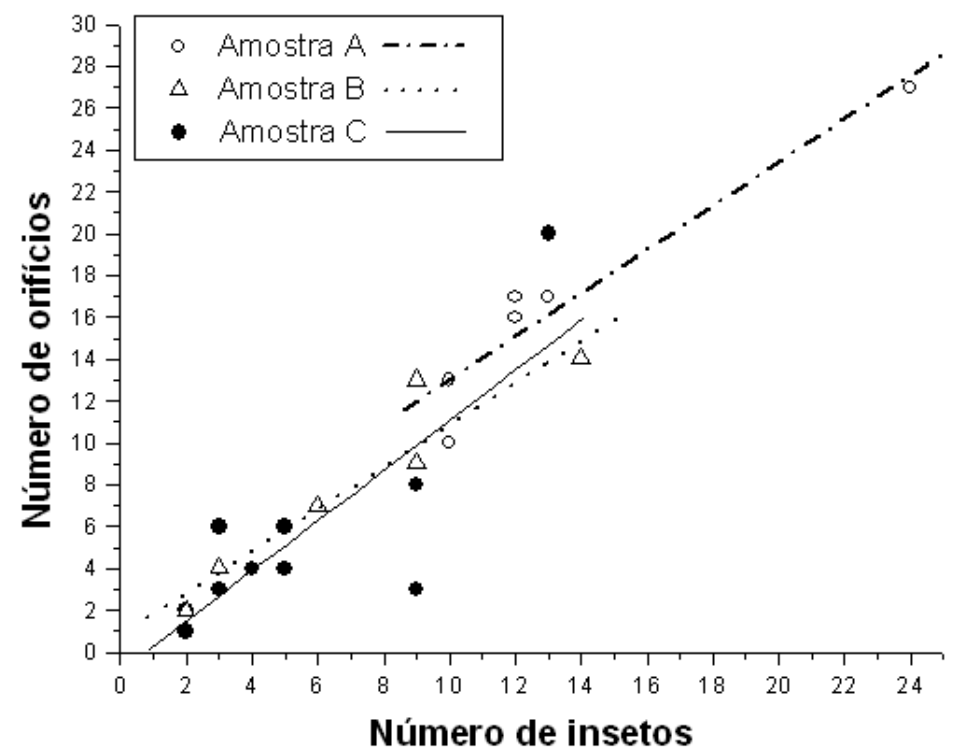

Figura 3. Correlação entre o número de insetos Pygiopachymerus lineola (Chevrolat, 1871) e o número de orifícios de saídas em de vagens de Cassia fistula (Leguminosae: Caesalpinoideae) em três bairros do município de Maceió,

Estado de Alagoas, no período junho a dezembro de 2006 (A, Jatiúca; B, Farol; C, Cidade Universitária).

Figure 3. Correlation between the number of insects Pygiopachymerus lineola (Chevrolat, 1871) and the number of holes in the fruit output of Cassia fistula (Leguminosae: Caesalpinoideae) in three districts of the city of Maceió,

Alagoas state, in the period from June to December 2006 (A, Jatiúca; B, Farol; C, Cidade Universitária). 
Considerando os frutos coletados de C. fistula em diferentes bairros, verificou-se que de 19 vagens da amostra A, $16(84,2 \%)$ continham ovos viáveis depositados na superfície das vagens; já na amostra B de 20 vagens todas apresentavam ovos viáveis; na amostra $\mathrm{C}$ das 18 vagens, $15(83,3 \%)$ possuíam ovos viáveis. Ribeiro-Costa e Costa (2002) obtiveram em posturas nas vagens maduras de $C$. leptophylla Vogel o valor de 97,77\%, confirmando assim a preferência da fêmea em ovipositar na superfície das vagens, demonstrando apenas 2,23\% posturas diretamente nas sementes.

As vagens foram coletadas com ovos na superfície, e em laboratório foi observado que os insetos fizeram orifícios circulares de saída demonstrando que $P$. lineola pertence ao grupo de bruquíneos que ovipositam diretamente na superfície das vagens.

Segundo Costa Lima (1955), os bruquíneos dividem-se em dois grupos quanto à realização da postura: os que põem os ovos nos frutos da planta hospedeira, acarretando o desenvolvimento das larvas nas sementes desses frutos atacados; e os que põem os ovos diretamente sobre a semente, quando esta se encontra separada do respectivo fruto, consequentemente, as larvas que deles eclodem penetram na semente e se desenvolvem. Sob o ponto de vista econômico, os bruquíneos são bastante importantes podendo se desenvolver continuamente nas sementes armazenadas. Esse fenômeno também foi confirmado por Johnson (1981).

A análise dos fatores bioecológicos de predação por $P$. lineola em vagens de $C$. fistula sugere um baixo nível de predação, demonstrando que a população deste bruquíneo pode não estar interferindo no controle dessa espécie leguminosa arbórea exótica, porém há necessidade de novas pesquisas mais aprofundadas aliadas a capacidade germinativa da planta. Nas amostras coletadas, a correlação foi alta mostrando a preferência das fêmeas desta espécie em ovipositar nas vagens do gênero Cassia L.

\section{AGRADECIMENTOS}

Os pesquisadores agradecem a Professora Dr. Cibele Stramare Ribeiro-Costa, Departamento de Zoologia da Universidade Federal do Paraná (UFPR), pela identificação de Pygiopachymerus lineola (Chevrolat, 1871). Ao Conselho Nacional de Desenvolvimento Científico e Tecnológico, pelo apoio e financiamento do projeto e à Universidade Federal de Alagoas pela disponibilidade da infraestrutura para realização dos trabalhos.

\section{REFERÊNCIAS}

ALVES, P. L. C. A.; PAVANI, M. C. M. D. Instruções básicas para a coleta e preparo de material botânico a ser herborizado. Jaboticabal, Funep, 1991.

ALMEIDA, L. M. de; RIBEIRO-COSTA, C. S.; MARINONI, L. Manual de coleta, conservação, montagem e identificação de insetos, $1^{\mathrm{a}}$. ed. Ribeirão Preto: Holos, Série Manuais Práticos em Biologia, 1998, 88 p.

BORN, F. S.; LIMA, I. M. M. Desenvolvimento pós-embrionário de Anteos menippe (Hübner) (Lepidoptera: Pie- ridae) em Cassia ferruginea Sharad. (Caesalpinaceae), em laboratório. Revista Brasileira de Entomologia, Curitiba, v. 49, n. 4, p. 522-526, 2005.

BRAGA, R. Plantas do Nordeste, especialmente do Ceará, $3^{\mathrm{a}}$. ed. Mossoró: Escola Superior de Agricultura de Mossoró, Coleção Mossoroense, v. 11, 1976. 256 p.

CARVALHO, G. A.; FIGUEIRA, K. L. Biologia de Pygiopachimerus lineola (Chevrolat, 1871) (Coleoptera: Bruchidae) em frutos de Cassia javanica L. (Leguminosae: Caesalpinioideae). Floresta e Ambiente, Seropédica, v. 6, n. 1, p. 83-87, 1999.

COSTA LIMA, A. Insetos do Brasil. 9. ${ }^{\circ}$ tomo, capítulo XXIX: coleópteros, 3. a parte. Rio de Janeiro: Escola Nacional de Agronomia, Série Didática n. 11, 1955, 289 p.

DANTAS, C. I.; SOUZA, C. M. C. Arborização urbana na cidade de Campina Grande - PB: Inventário e suas espécies. Revista de Biologia e Ciências da Terra, Campina Grande, v. 4, n. 2, p. 1-19, 2004.

JANZEN, D. H. Escape of Cassia grandis L. beans from predators in time and space. Ecology, Ithaca, v. 52, n. 6, p. 964-979, 1971.

JOHNSON, C. D. Interactions between bruchid (Coleoptera) feeding guilds and behavioral patterns of pods of the Leguminosae. Environmental Entomology, Lanham, v. 10, n. 2, p. 249-253, 1981.

LIMA, C. B.; BELLETTINI, N. M. T.; SILVA, A. S.; JANANI, J. K.; AMADOR, T. S.; VIEIRA, M. A. V.; CHEIRUBIM, A. P. Descrição das árvores encontradas nas ruas de Bandeirantes-PR. Revista Brasileira de Biociências, Porto Alegre, v. 5, n. 1, p. 609-611, 2007.

LORENZI, H. Árvores brasileiras: Manual de identificação e cultivo de plantas arbóreas nativas do Brasil. Nova Odessa: Ed. Plantarum, 2001, 1088 p.

LORENZI, H. Chave de Identificação - para as principais famílias de Angiospermas nativas e cultivadas do Brasil. São Paulo: Plantarum, 2007.

LOUREIRO, M. B.; CARVAlHO, A. G.; ROSETTO, C. A. V. Danos causados por insetos à germinação e no vigor de sementes de Apuleia leiocarpa Vog. Macbride. Agronomia, Seropédica, v. 38, n. 1, p. 105-109, 2004.

MATTIUZ, C. F. M.; CAMPOS, L. Z. O.; PINTO, A. S. Levantamento de plantas ornamentais e cochonilhas associadas em residências de Ribeirão Preto (SP). Revista Brasileira de Horticultura Ornamental, v. 12, n. 1, p. 43-51, 2006.

MISSOURI Botanical Garden - Tropicos. Disponível em $<$ www.tropicos.org $>$ 
PEREIRA, M. C. D.; SANTOS, D. B.; FILHO, F. F.; MELO, M. D. Levantamento Florístico do conjunto dos professores, Natal/Rn: um subsídio para Arborização Urbana. Revista Cultural e Científica Carpe Diem, v. 8, n. 8, 2010.

SARI, L. T.; RIBEIRO-COSTA, C. S.; ROPER, J. J. Dinâmica populacional de bruquíneos (Coleoptera, Chrysomelidae) em Senna multijuga (Rich.) H. S. Irwin \& Barneby (Caesalpinaceae). Revista Brasileira de Zoologia, v. 22, n. 1, p. 169-174, 2005.

SANTOS, G. P.; ZANUNCIO, J. C.; ANJOS, N.; JÚNIOR S. L. A. Danos causados por insetos a sementes de Apuleia leiocarpa (Leguminosae:Caesalpinioideae). In: Anais da Sociedade Entomológica do Brasil, Itabuna, v. 18, n. 2, p. 257-266, 1989.
RIBEIRO-COSTA, C. S.; COSTA, A. S. Comportamento de oviposição de Bruchidae (Coleoptera) predadores de sementes de Cassia leptophylla Vogel (Caesalpiaceae), morfologia dos ovos e descrição de uma nova espécie. Revista Brasileira de Zoologia. v. 19, n. 1, p. 305-316, 2002.

RICKLEFS, R. E. A Economia Da Natureza:Um Livro Texto em Ecologia Básica. $5^{\text {a }}$ ed. Editora Guanabara KOOGAN S.A. Rio de Janeiro, 2001, 470p. 